This is the latest paper in a series of Injury Classics. Our goal is to reprint one or two such papers in each issue to introduce newcomers to these old, often quoted, and important contributions. As many are difficult to find, it should help all of us to have a copy at hand. Your suggestions about future articles are welcome. Write to the editor with details of your favourite, most quoted paper.
Dr Haddon is Director, National Highway Safety Bureau, Federal Highway Administration, US

Department of

Transportation,

Washington, DC 20591.

This paper first appeared in the American fournal of Public Health (1968;58:1431-8) Health (1968;58:1431-8)
and is reproduced with permission. Copyright 1968 by the American Public Health Association.

\title{
The changing approach to the epidemiology, prevention, and amelioration of trauma: the transition to approaches etiologically rather than descriptively based
}

\author{
William Haddon Jr
}

Phenomena of trauma to be dealt with scientifically must be based not on descriptive categorizations, but on etiologic ones. How this is happening and what it means are discussed in this paper.

\section{Background}

Approaches to the phenomena of trauma, which are of interest here, are rapidly becoming more rational and scientific. None the less, the field still includes the only substantial, remaining categories of human morbidity and mortality still viewed by most laymen and professionals alike in essentially prescientific terms. The traditional wisdom perpetuates terms and concepts formerly applied to much of human experience. "Luck", "chance", "accident", and other extrarational notions still survive from the times when scientific explanations for plagues, earthquakes, "natural disasters", and other terrifying phenomena scourged a mankind that had no rational understanding, either of their sources or of the means for dealing with them.

Unfortunately, because of their automatic subscription to the traditional, prescientific wisdom of the field, many professionalsphysicians, behavioral scientists, and others-in coming to this field for the first time, still merely translate the traditional wisdom and its terms into their own scientific framework and jargon. Building on the result, they assume they have contributed something. This, however, is likely to occur decreasingly because of the accelerating transition in concepts and research now taking place.

What then, is the essence of this transition? Very broadly and importantly, it is part of the increasing awareness of the relationships between man and his environment, of human ecology, especially of man's relationships with certain potentially or actually hazardous physical and chemical attributes of his environment.

At the beginning of the nineteenth century, man was equally ignorant of both the physical and chemical hazards of his environment, on the one hand, and of the biological hazards on the other. In the 150 years that followed, he moved disproportionately rapidly in unravel- ling and controlling the biological hazards, but is only now beginning on the physical and chemical hazards which range from air pollutants to the forces at play on our highways.

The accompanying transition in categorizations of the phenomena of the field has many precedents in medicine. It is the shift from descriptive thinking and nosology to categorizations in etiologic terms. In the past, this shift has almost invariably been accompanied by increasingly successful control efforts, as one would expect, because ability to describe human morbidity and mortality etiologically requires an understanding of causation. Hence it opens the door to the possibility of manipulation and control.

What are a few past examples of this routine transition from descriptive categorizations or diagnoses to those etiologically based? In illustration, such notions as fever and wasting were once diseases. As the evolving science of the past was applied, it did not, however, find single causes of these, substituting causally based classifications on a one-to-one basis for the earlier terms and the ideas with which the phenomena were described. Rather, new groupings etiologically based were constructed that picked up portions of the earlier, descriptive groupings or sets, as illustrated in fig 1 . Viewed differently, for example, the set of phenomena

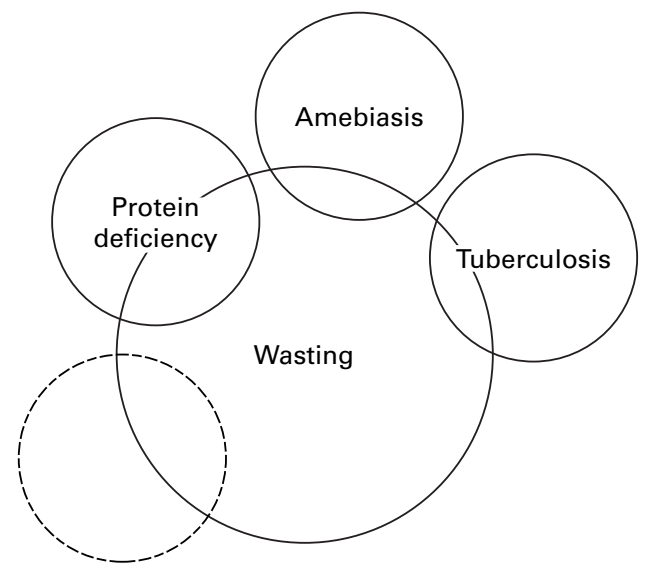

Figure 1 An illustration of the parcelling out to etiologically defined sets of the components of a descriptively defined set of pathology. 


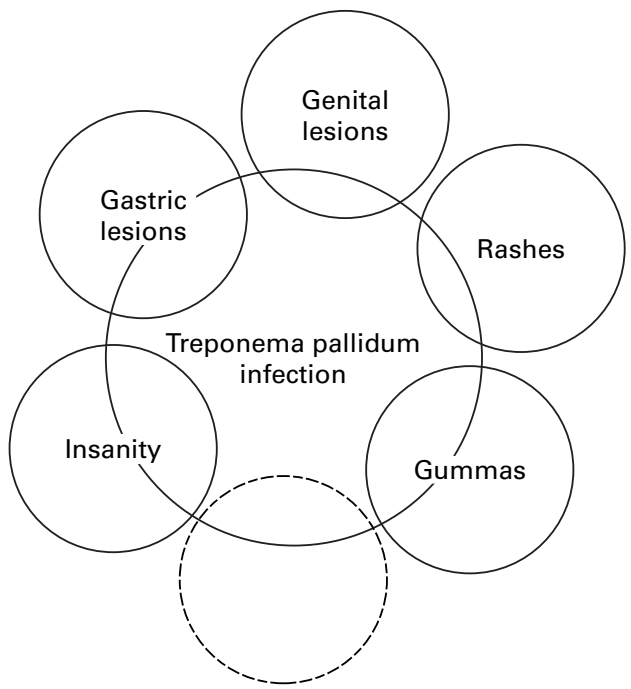

Figure 2 An illustration of the incorporation of portions of various descriptively defined sets of pathology into an etiologically defined set.

formerly referred to as "wasting", was parcelled out to such etiologic sets as tuberculosis, amebiasis, and a host of others.

More relevant for our purposes here is to view the process in reverse; that is, from the standpoint of the etiologic sets in picking up pieces of many pre-existing descriptive sets, as illustrated in fig 2 .

Thus, syphilis, the etiologic set based on the infectious agent, Treponema pallidum, picked up parts of previous descriptive sets, such as paresis, gummas, penile lesions, rashes, certain gastric lesions, certain abnormalities of the growing ends of bone, and many others, but not all of those in any one of the earlier descriptive sets. Again, an important point is that there is almost never in such transitions in nosology a one-to-one relationship between the earlier, descriptive ways of looking at the phenomena and those etiologically based which are substituted for them.

\section{Concepts, old and new}

Those who do not understand this typical and longstanding pattern of transition usually refuse new etiologic categorizations and evidence because they maintain, correctly, that these do not account for all of the descriptive categories involved, or for all of the problems associated with them. This commonplace failure to understand this usual, and necessary, transition has, incidentally, especially been a problem in connection with research on descriptively based groupings such as "atherosclerosis", "chronic bronchitis", "cancer", "diabetes", and many others of current concern. This failure in understanding has also delayed research and programs concerned with what most still refer to as "accidents".

The notion of an accident is descriptive, not etiologic. It has a long history and close relationship - themselves worthy of the most careful study by behavioral scientists-to notions of personal vulnerability and invulnerability. This is illustrated, for example, by the themes of the stories of Job and Achilles.
Redolent of the extrarational and supernatural and prescientific, they remind one of $\mathrm{Ma}-$ linowski's natives in their approach to the hazards outside the reef, which they did not understand, in comparison with those inside which they approached in more rational terms. ${ }^{1}$

But the old, descriptive, concept of an accident is largely giving way in programs and research to an etiologic concept which, in turn-as is typical in such transitions-is picking up a number of phenomena not normally included under the term accident itself. As with the examples I have briefly sketched, neither set includes all of the ingredients of the other.

The etiologic basis is the various forms of energy exchange which must occur in excess of body injury threshold, for the injuries which make the field of such current social concern to occur. The forms of energy involved in producing so-called "accidental injuries" of all types, and without which they cannot occur, include thermal energy, ionizing radiation, mechanical energy, electrical energy, and chemical energy, each in a variety of forms. Since these have been discussed in detail elsewhere, ${ }^{23}$ we need not go into them further here, except to note that in the highway safety area the problem is almost exclusively one of mechanical energy reaching people at rates that involve forces in excess of their injury thresholds. None the less, some chemical and thermal hazards appear to be of increasing importance on the highway; for example, in relation to the distribution of hazardous amounts of propane, explosives, and other hazardous materials, on our roads.

The literature in the overall field is increasingly divergent. Most of the behavioral science literature is highly inbred and concerned almost exclusively with studies and programs centering on the use of the descriptive notion of "accident". Parallel to this literature is another-substantially based in physics and medicine - already very extensive and growing much more rapidly, and largely derived from De Haven's 1942 landmark paper entitled "Mechanical analysis of survival in falls from heights of fifty to one hundred and fifty feet". ${ }^{4}$ This second literature, incidentally, is already producing major payoffs in injury prevention and amelioration. It is concerned with the forces that produce the injuries to animate and inanimate structures, the ways these can be avoided, the susceptibilities of the animate and inanimate structures involved, and the amelioration of the various forms of damage, once they occur.

An outstanding exception to the preoccupation of the behavioral literature with "accidents" is James J Gibson's 1961 paper, "The contribution of experimental psychology to the formulation of the problem of safety-a brief for basic research". ${ }^{5}$ Gibson's subsections delineate an ecological and etiological approach: "a classification of dangers" (wherein he discusses, for example, the various forms of energy thermal, radiant, chemical, electrical); "exploratory behavior and margins of safety" (that is, failure to perceive, failure to react, the 
concept of an accident, and the role of motivation); and "experimental research relevant to perceiving and avoiding dangers". Here the author's approach is solidly on the scientific side of the issues, rather than to translate into scientific terms essentially prescientific and extrarational notions. This, in my opinion, is clearly the direction that most behavioral research should and will take.

However, the former still prevalent notion of an "accident" should continue to serve as a basis of some research because it is so ingrained in our culture. Unless we learn the ramifications of this notion, we will not know how the behaviors to which it relates can be manipulated. This is probably a passing opportunity and one of great potential value, especially in relation to ideas of personal invulnerability, subjective probability, and many others. In shifting, none the less, in Gibson's direction, research should concentrate on the much larger issues involved in the etiologic set since they include concepts related to hazards of all kinds, not only physical and chemical, but also whether expected or not. Moreover, the limitation should not be to civilian phenomena alone.

Control opportunities, in parallel with the handling of problems posed by other environmental hazards in the past, must be solidly based in relation to etiologic organization of the field, not on descriptive categorizations. Although I have elsewhere spelled out a much more detailed analysis of ways one can analyze the problems of physical and chemical hazards, and the prevention of harmful interactions with them, ${ }^{23}$ here are a few examples illustrating, with concepts compatible with Gibson's the utility of this approach.

It is the end results, again, of structural damage and death that make the problem of social concern, and it is these that must be reduced. The problem thus is not a priori "to prevent accidents" per se. In the case of the very parallel situation, where the social objective was to reduce the end result of epidemic paralysis, this would have been equivalent to preventing infection. However, as we subsequently learned, preventing infection was not the only or best way to prevent the end result of injury and death due to the virus in question.

\section{Phases of social concern}

Rather, as with polio, there are essentially three major portions or phases of the sequence of events leading up to the end results, during which causal factors are active and countermeasures can be undertaken.

The first phase involves, from a countermeasure standpoint, the prevention of the etiologic agent from reaching the susceptible host. In polio, this used to involve keeping children out of swimming pools and from attending the movies. In the highway field, this involves an array of measures which, in essence, are designed to prevent mechanical forces above injury thresholds from reaching vehicles and people.
A second phase in the interactions, which lead up to the end results of social concern, involves the interaction of the etiologic agents and the susceptible structures. In the case of polio, this begins with the arrival in the host of the virus and involves its interactions with the cells of his intestinal tract, and, later, of his central nervous system. In the case of highway phenomena, it begins when mechanical forces, in excess of those the vehicle, occupants, pedestrians, and cyclists can tolerate, begin to exert themselves on vehicles and people. Here, too, are many opportunities for the elimination or reduction of the end results of injury and death. In fact, as far as vehicle occupants are concerned, the knowledge, both theoretical and applied, is already available. During the next few years, this will make it entirely feasible to design vehicles whose occupants need not sustain either any injuries at all or, at worst, no very serious injuries at crash speeds under at least 60 miles an hour, a range in which the overwhelming bulk of occupant injuries and deaths now occur. (In illustration, Bohlin ${ }^{6}$ has recently shown reductions in deaths in highway crashes, other factors being equal, of $80 \%$ as the result of the use of combination lap-and-upper-torso safety belts. In addition, no deaths occurred in his series below 60 miles per hour, compared with 12 miles per hour among the unbelted. ${ }^{6}$ ) Without going into further details, the methods applied will, in essence, involve the far better "packaging" of human cargo, accomplishing with people what we long ago achieved with property. $^{23}$

A third phase of the sequences, which lead up to the end results of concern, involves maximizing salvage, once damage has been done to the susceptible structures. Using polio again as an example, although there are many others in the medical field, the problem there was to reduce the likelihood of death, once paralysis occurred, to lower the extent and progression of paralysis, to reverse it insofar as possible, and to provide the necessary emergency medical, intermediate, and rehabilitative care. Needless to say, the timing and sequencing of salvage and a good deal of its substance are identical to those appropriate for those injured on our highways.

Clearly, the questions and opportunities, at each point in these etiological sequences, are different from those likely to be recognized by merely talking about injury control or about "accidents", using the old and still traditional nosology and its penumbra of folklore and traditional wisdom.

\section{Technics in use}

For simplicity, most of the problems of the field can be sorted out with a two dimensional matrix, within the cells of which one can organize a great many more specific issues. Figure 3 shows this as done for the highway portion of the overall chemical and physical injury problem. Similar matrices are used for other portions of the overall problem. Doing so is also an important step in sorting out interactions between the items covered in the 


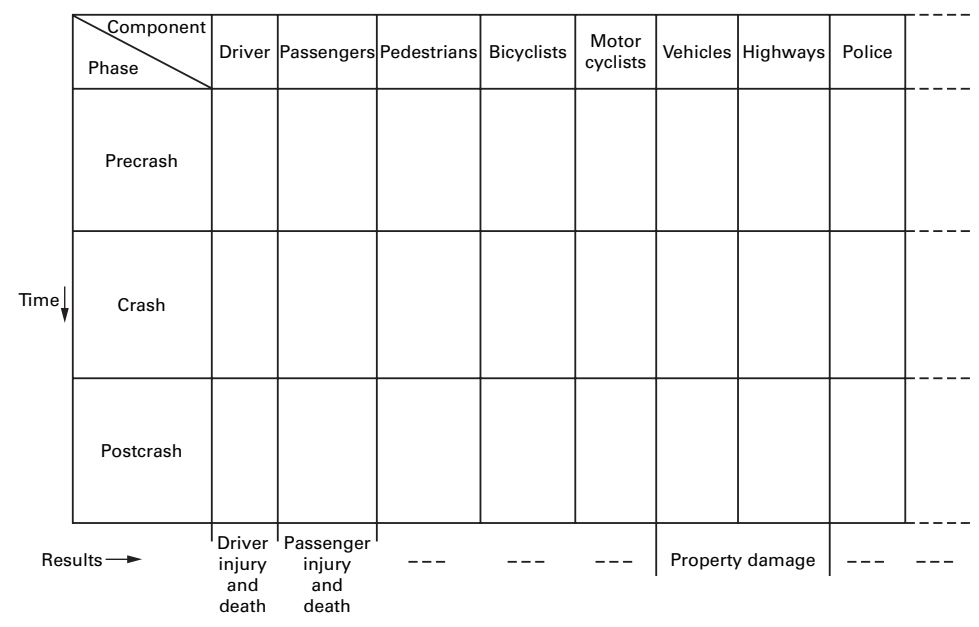

Figure 3 A matrix for identifying major areas within the highway portion of the overall chemical and physical injury problem.

individual cells and for purposes of mathematical modeling. Each of these cells contains a substantial number, breadth, and complexity of factors, categories of variables, and opportunities for influencing the end results.

If fully developed here, two analogous matrices would be set up: the first concerned with causal factors in each cell; the second with countermeasures in each. We also use this matrix to identify resource requirements and what is known scientifically.

It should be noted that this matrix identifies the location of all, as far as we know, that is done in the field to reduce the end results of concern. It is much broader than preoccupation with the causation of accidents (that is, crashes) and their prevention. For example, in the precrash phase, the research issues include those of alcoholism, blowouts, coefficients of friction of road surfaces. In the crash phase, there are the injury thresholds of drivers and others, the dynamic integrity of vehicle "packages", and highway crash design. In the postcrash phase, the issues for research and programs include emergency signal generation and other communications, emergency transportation, emergency medical care, debris removal, and police work.

The most common and universal fallacy in the field, whether viewed within a descriptive or etiologic framework, is one which is so ingrained that it is seldom explicitly recognized. It involves the assumption that the priority rank of countermeasures in terms of their ability to influence the end results of concern, must parallel the ranking, in order of their relative contributions, of causes influencing those end results. In its most common form, it states that because drivers cause most accidents, programs correspondingly must be concerned with drivers. In the real world, there is no basis for making this assumption, especially since in numerous areas of the field it leads to demonstrably false conclusions.

Thus, if applied to the widespread thermal and electrical injuries associated with early house wiring systems, this theory would have led to concentrating on attempts to influence human behavior rather than the development of the fuse. With respect to motorcycle accidents, where we know that compelling the use of appropriate helmets reduces deaths and injuries about $65 \%$, it would have us concentrate, despite our present lack of knowledge of ways to influence crashes, only on the present far less productive manipulation of motorcycle drivers. ${ }^{2}$ In the case of occupant protection, which we know has great potential, ${ }^{2}$ some of it already beginning to be realized, this fallacy has set back for many years the application of such information because of its insistence that the driver was the problem and, therefore, should be, in essence, the only locus of countermeasure activity.

Earlier it was emphasized that transitions from descriptive to etiologic groupings of phenomena do not occur on a one-to-one basis. It has been mentioned that the notion "accident" includes a number of things not directly relevant to highway or other injuries and deaths. It includes the entire area of "mishaps", with all their extrarational and other overlays, all of which should be systematically explored by behavioral scientists and other research workers.

Similarly, groupings of phenomena based on the kinds of injurious energy exchanges involved, for example, those defined in terms of mechanical energy, include a number of phenomena not now considered under the term "accident". Some of these should be of great scientific value, if explored in behavioral and other terms. These, in turn, are quickly discovered to have many things in common with problems related to the more traditional highway and other "accidents".

For example, within the highway area, this broader approach, based on the etiologic agents which must be involved, picks up in the first phase ${ }^{\star}$ the occasional murders and suicides which many of us working in this field have occasionally come across. The approach also picks up a number of equivalents that involve individual and organized violence and aggression, whether on the civilian scene or in war. Thus, one can identify for study, across human violence, the roles of alcohol, views of risk, notions of individual invulnerability, and subjective probability. ${ }^{3}$

As another example, in the second phase, body tolerance to mechanical injury is the same, regardless of whether the body is subjected to mechanical forces deliberately or inadvertently on the highway or in the jungle. Similarly, countermeasures, too, are soon discovered to have many common denominators, whether they involve, for example, the use of military and police helmets, or helmets worn by motorcyclists or the interior padding of windshield header areas in automobiles. Parallel points can be made, of course, for a number of the behavioral aspects of all of these and related problems.

Similarly, in the third phase, involving salvage after the injuries have occurred, the problems

*In the highway "safety" field, these phases are termed the "precrash", "crash", and "postcrash" phases, respectively. 
related to the factors that aggravate or increase the success of salvage are essentially identical, whether the issue is injury on the highway or in jungle warfare. The injuries, the measures that need to be taken, and the time constraints are all virtually identical. The response involves prompt command and control, communications, and transportation, with all of the opportunities for research and the application of research findings that this embraces.

Also, it should he noted that in the past we have ambiguously separated and treated, in conceptually different terms, the acute interactions of man and his structures with environmental hazards from those operating over longer periods, even though the two in many cases have identical results. Thus, for example, if sulphur dioxide acutely damages man, we have traditionally viewed the interaction descriptively and prescientifically as an "accident" and emphasized the unplanned nature of the event and its chance aspects, not the nature of the agent and the means by which it reached or could have been prevented from reaching the susceptible host. To the contrary, even though the end result might be biologically identical, the interaction involving the same agent, arriving over a longer period of time in smaller doses, has been regarded in terms of traditional preventive medicine concepts. These emphasize the nature of the agent and the ways to prevent either its generation, its release, or its arrival at the person or group to be protected. Here we have talked in terms of maximum permissible concentrations in the working environment and in the air we breathe in our cities, not in terms of chance. By emphasizing the nature of the injurious etiologic agents, involved in the former "accident" area, we eliminate this logical inconsistency and open the door for success in control.

\section{Conclusion}

It must be emphasized again that it is essential that those who would make scientific contributions in this field avoid approaches which, in effect, translate the traditional, prescientific wisdom into scientific terms and jargon. We must recognize the conceptual transition in which we are participating and the difference between descriptive and etiologic approaches, and that the payoffs for society and in scientific understanding lie chiefly in the latter. We must also recognize that this area and approach must be considered as part of any overall approach to human ecosystems, and that we can no longer afford to deal in the terms and concepts of the past, with their vague emphasis on threatening forces. Such an approach is more appropriate for primitive tribes than for modern society.

This paper was presented before the Epidemiology Section of the American Public Health Association at the Ninety Fifth Annual Meeting in Miami Beach, Florida, 26 October 1967

1 Malinowski B. Magic, science and religion, and other essays. Garden City, NY: Doubleday (Anchor Books), 1954.

2 Haddon W Jr. The prevention of accidents. In: Clark and MacMahon, eds. Textbook of preventive medicine. Boston, MA: Little Brown, 1967 (chapter 33).

3 Haddon W Jr, Suchman, EA, Klein D. Accident research. Methods and approaches. New York: Harper \& Row, 1964.

4 De Haven, H. Mechanical analysis of survival in falls from heights of fifty to one hundred and fifty feet. War Med 1942;2:586-96 (July).

5 Gibson JJ. The contribution of experimental psychology to the formulation of the problem of safety-a brief for basic the formulation of the problem of

6 Bohlin NI. A statistical analysis of 28000 accident cases with emphasis on occupant restraint value. Proceedings of with emphasis on occupant restraint value. Proceedings of the 11 th Stapp Car Crash Conference, 10-11 Oct 1967.
New York: Society of Automative Engineers Inc, 1967.

\title{
SPECIAL REPORT
}

\section{Inequalities in health}

\author{
Carol Sherriff
}

Child Accident Prevention Trust, 4th Floor, Clerks Court, 18-20 Farringdon Lane, London EC1R 3HA, UK

Correspondence to: Carol Sherriff (e-mail: carol@capt.demon.co.uk).
In July 1997, shortly after the election of the new Labour government in the UK, the Secretary of State for Health asked Sir Donald Acheson, the recently retired Chief Medical Officer, to head an inquiry into inequalities in health in England and identify priority areas for policies to reduce inequalities.

Sir Donald Acheson's report adopts a broad socioeconomic model of health and inequalities. ${ }^{1}$ This model places individuals at the centre of many layers of conditions that determine their health, for example, their lifestyle, social and community networks, living conditions, and general socioeconomic conditions. As well as examining physical and mental health, the inquiry team sought evidence of the effects of unemployment, low income, housing, transport, and education.

There are two overarching recommendations. First, that as part of health impact assessments, all policies likely to have a direct or indirect effect on health should be evaluated in terms of their impact on health inequalities. They should be formulated in such a way that by favouring the less well off they will, wherever possible, reduce inequalities. Mechanisms to 
monitor progress should be developed and better data collected to evaluate the effectiveness of these policies. Second, a high priority should be given to policies aimed at improving health and reducing inequalities in women of childbearing age, expectant mothers, and young children.

The report goes on to make a wide range of recommendations. In relation to reducing inequalities in childhood injury it states that more should be done to improve housing, particularly temporary accommodation, to encourage walking and cycling and reduce traffic speed, and to provide social and emotional support for parents and children.

In addition to these specific recommendations, the inquiry team also recommended that the needs of minority ethnic groups be specifically considered in the development and implementation of policies aimed at reducing inequalities. They called for a more appropriate allocation of resources to take account of the needs of communities at greatest risk.

There is also a plea for policies to develop the capacity for tackling health inequalities. For example, it recommends that directors of public health produce an equity profile for the population they serve, and undertake a triennial audit of progress towards achieving objectives to reduce inequalities in health. It suggests that there should be a duty of partnership between the health service and regional government to ensure that effective local partnerships are established between health, local authorities, and other agencies and that joint programmes to address health inequalities are in place and monitored.

Sadly, the section that details current inequalities deals mainly with mortality and morbidity rates of adults. Little is said about inequality in childhood injury. Yet accidental injuries have the steepest social gradient of any form of death in children and young people. The recommendations are in the main rather vague and many simply recommend further development of existing strategies. However, these disappointments should not conceal the powerful impact this report is likely to have. If all housing, environmental, transport, and educational policies in England as part of their health impact assessment have to take into account the health impact on children living in poorer families, we could see substantial changes to those policies. Moreover, the forthcoming government white paper on public health is likely to set new targets for reducing childhood injury and inequalities in health. This will add impetus to the implementation of the recommendations.

This raises a number of challenges for the Child Accident Prevention Trust and other organisations committed to reducing childhood injury. Although we may criticise the report for being thin on detail about inequalities in childhood injury, we have not successfully made the case for it to be the cornerstone of any strategy to reduce inequalities in health. We have not yet shown, although we believe it to be so, that childhood injury leads to high levels of permanent disabilities, lowered educational attainment, emotional distress, family break-up, and long term economic hardship. We do not know whether injury sustained in childhood leads to physical or emotional health problems in later life. It is also not clear which interventions are effective in reducing inequalities. Interestingly, a recent trend analysis of socioeconomic differentials in deaths from injury in childhood in Scotland suggests that growth in inequalities may not be an UK-wide phenomenon. ${ }^{2}$ The authors of the analysis are not sure of the reasons for this difference but put forward three possible explanations. One is that injury prevention measures have an equal effect in Scotland; another that the decline occurred independently of prevention efforts. Or it may be that different measures of inequality (area based in Scotland as opposed to individually based in England) yield different results.

Whatever the explanation, tackling inequalities in childhood injury presents a major challenge for government, academic researchers, and organisations committed to implementing effective prevention strategies.

1 Acheson D, chairman. Independent inquiry into inequalities in health. London: The Stationery Office, 1998

2 Morrison A, Stone DH, Redpath A, et al. Trend analysis of socioeconomic differentials in deaths from injury in childhood in Scotland, 1981-95. BMF 1999;318:567-8. 